\title{
Practical tracking control of piezoelectric actuators with time-delay estimation and nonsingular terminal sliding mode
}

\author{
Haiping Lin ${ }^{1}$, Hanlie $\mathrm{Gu}^{2}$, Jinyu $\mathrm{Ma}^{3,4,}{ }^{*}$, and Shengdong $\mathrm{Yu}^{4}$ \\ ${ }^{1}$ Hangzhou Vocational and Technical College, Hangzhou 310018, China \\ ${ }^{2}$ Aerospace System Engineering Shanghai, Shanghai 200000, China \\ ${ }^{3}$ Nanjing University of Aeronautics and Astronautics, Nanjing 210016, China \\ ${ }^{4}$ Wenzhou Polytechnic, Wenzhou 325000, China
}

\begin{abstract}
A novel type of nonlinear robust control strategy is proposed in view of uncertain nonlinear factors, such as hysteresis, creep, and highfrequency vibration, of piezoelectric actuators (PEAs). This strategy can be used for the precise trajectory tracking of PEAs. The Bouc-Wen dynamic model is reasonably simplified to facilitate engineering application. The hysteresis term is summarized as an unknown term to avoid its nonlinear parameter identification. The controller robustness is achieved due to the nonsingular terminal sliding mode control, and the online estimation of unknown disturbances is realized because of the delay estimation technology; thus, no prior knowledge of the unknown boundary of the system is required. The precision robust differentiator is used to estimate the speed and acceleration signals in real time on the basis of the obtained displacement signals. The closed-loop stability of the system is proved by the Lyapunov criterion. Experimental results show that the proposed control strategy performs better than the traditional time-delay estimation control in terms of control accuracy and energy conservation. Therefore, the proposed control strategy can play an important role in the micro/nanofield driven by PEAs.
\end{abstract}

Keywords: Piezoelectric actuators, Sliding mode control, Trajectory tracking, Time delay estimation.

\section{Introduction}

In recent years, the high-precision micro/nano-operation technology has played an increasingly important role in modern industrial systems [1]. Piezoelectric actuators (PEAs), such as micromanipulators [2], atomic force microscopes [3], MEMS manufacturing [4], micro-operation robot [5], and intracytoplasmic sperm injection [6], are widely used in micro/nano-operations due to their advantages (e.g., large output force, high resolution, and high response frequency) [7]. However, uncertain nonlinear factors, such as hysteresis,

*Corresponding author: jinyuma@,nuaa.edu.cn 
creep, and high-frequency vibration, severely restrict the application of PEAs [8]. Therefore, appropriate control strategies for motion control are necessary to overcome the displacement error produced by uncertain nonlinear factors and achieve fast response of PEAs and high-precision trajectory tracking. In modern control theory, a robust controller can be constructed on the basis of the feedforward, feedback, or composite control [9]. Feedforward control achieves compensation by establishing the hysteresis model of PEAs. Many scholars exhibit their own unique achievements in the establishment of PEA hysteresis model [10-14]. However, the dynamic model of PEAs is nonlinear with a complex structure and numerous parameters. Parameter identification is a tedious and timeconsuming task. Therefore, feedforward or complicated control is not conducive to engineering applications. A feasible approach is to implement accurate estimation and compensation of unknown disturbances and suppress the influence of uncertain nonlinear factors on the control accuracy by designing a robust closed-loop controller and regarding the uncertain nonlinear factor as an unknown disturbance. Accordingly, precise trajectory tracking is achieved, and PEAs will have high engineering applicability in micro/nanooperations.

Sliding mode control is one of the important discoveries in modern control theory and is an effective tool for solving nonlinear system control problems [15]. Sliding mode control is insensitive to model uncertainty, parameter changes, and external disturbances [16]. Terminal sliding mode (TSM) [17] is proposed to improve the convergence speed of the sliding mode control. The convergence speed of TSM exceeds that of the traditional sliding mode based on linear hyperplane. TSM is widely used in linear or nonlinear systems with uncertain factors. However, singularity problems exist in the TSM method. A nonsingular TSM (NTSM) [18] is proposed to avoid nonsingularity problems. Therefore, a robust control by NTSM must be achieved given that unknown terms exist in the dynamic model.

The time-delay estimation (TDE) technology, which was first proposed by Jin [19], can be used to achieve a model-free control. The TDE aims to estimate the unknown dynamics and perturbations at the previous moment through time-delay information. Therefore, prior knowledge of the unknown quantity boundary is unnecessary. The TDE technology can be applied to online estimation and compensation of unknown terms [20]. However, only a displacement sensor is often provided in actual engineering because the acceleration signal of the previous moment needs to be collected in the implementation of TDE. The mathematical and physical meaning of acceleration signal is the second differentiation of the displacement signal, which exacerbates the "differential explosion of noise signals". Therefore, a robust exact differentiator (RED) [21] is applied in reducing noise interference and obtaining accurate speed and acceleration signals.

This study aims to explore a sliding-mode controller that can be easily implemented by a computer, has a simple structure, and nonsingular. The main contributions are summarized as follows: 1) The Bouc-Wen dynamic model is appropriately simplified, and the hysteresis term is summarized as an unknown term that is beneficial to engineering application. 2) The TDE is used to achieve real-time estimation and compensation of unknown quantity to ensure that the controller is not required to obtain the boundary of an unknown quantity. 3) The combination of the NTSM, TDE, and RED technologies can obtain model-based, high-precision, and robust control effects.

The rest of this article is organized as follows. Section 2 simplifies the Bouc-Wen kinetic model of PEAs. Sections 3 designs a nonlinear robust controller, and Section 4 proves the stability of the closed-loop system. Section 5 carries out computer simulation experiments, and Section 6 performs hardware-in-loop simulation experiments on the controller. Section 7 concludes the paper. 


\section{System description}

\subsection{Problem description}

The open-loop experiment on PEAs reveals the relationship between the input voltage and the output displacement. A sinusoidal voltage with variable frequency and amplitude (Figure 1(a)) is applied to PEAs to obtain a voltage displacement curve (Figure 1(b)). The voltage and displacement are nonlinear with a strong hysteresis effect: the different voltage frequencies and amplitudes correspond to various hysteresis loops.

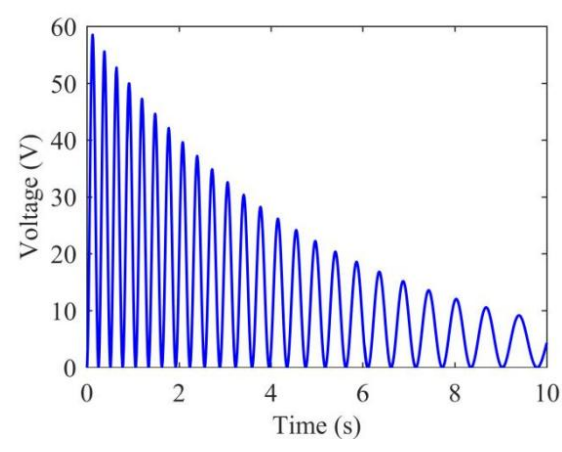

(a)

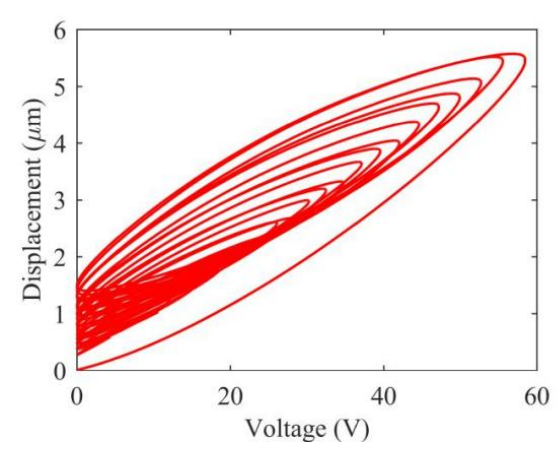

(b)

Fig. 1. Nonlinear hysteresis effect of PEAs. (a) Input voltage and (b) voltage displacement curves.

\subsection{Dynamic equation}

Dynamic equation of stacked PEAs is written according to the Bouc-Wen model [14]:

$$
\begin{gathered}
m \ddot{x}+b \dot{x}+k_{e} x=k_{e}(d u-h)+f_{d} \\
\dot{h}=\xi_{1} d \dot{u}-\xi_{2}|\dot{u}| h-\xi_{3} \dot{u}|h|
\end{gathered}
$$

where parameters $m, b$, and $k_{e}$ stand for equivalent mass, damping coefficient, and stiffness coefficient, respectively; $x, d$, and $u$ denote the displacement, piezoelectric coefficient, and input voltage, respectively; $f_{d}$ is used to describe the total disturbance of the unmodeled terms and external interference; $h$ is the hysteresis effect of the system; and $\xi_{1}, \xi_{2}, \xi_{3}$ is the hysteresis coefficient that describes the loop shape. The input voltage and its derivative pose a direct influence on the hysteresis effect. The dynamic model and parameter structure of PEAs are highly complex, and the parameter identification process is tedious and time consuming. A dynamic model with sufficient accuracy is nearly impossible to obtain due to the existence of an external interference. Therefore, a reasonable optimization of the Bouc-Wen model will be helpful to the design of a robust controller. 


\section{Design of a nonlinear robust controller}

\subsection{Controller design}

The controller is designed to achieve an exact tracking of actual displacement $\mathrm{x}$ to expected displacement $x_{d}$. Therefore, the displacement, velocity, and acceleration errors can be expressed as $e=x_{d}-x, \dot{e}=\dot{x}_{d}-\dot{x}$ and $\ddot{e}=\ddot{x}_{d}-\ddot{x}$, respectively.

The total nonlinear disturbance terms, including the hysteresis effect and external disturbance, are defined as unknown terms $(\Delta P)$ :

$$
\Delta P=-\frac{k_{e} h}{m}+\frac{f_{d}}{m} .
$$

Dynamic equation (1) is rewritten as follows:

$$
\ddot{x}=\frac{k d}{m} u-\frac{b}{m} \dot{x}-\frac{k}{m} x+\Delta P .
$$

The nonsingular terminal sliding mode surface is adopted [15]

$$
s=e+K \dot{e}^{p / q},
$$

where $K$ is the parameter to be adjusted; $p$ and $q$ are positive parameters; and $1<p / q<2$.

The input of the control law can be designed as follows:

$$
u=\frac{m}{k_{e} d}\left(\ddot{x}_{d}+\frac{q}{p} K^{-1_{\dot{e}}^{2} 2-p / q}+K_{s w} \operatorname{sgn}(s)\right)+\frac{b}{k_{e} d} x+\frac{1}{d} x-\frac{m}{k_{e} d} \Delta \hat{P},
$$

where $\operatorname{sgn}(\mathrm{s})$ is a sign function, and $K_{s w} \operatorname{sgn}(\mathrm{s})$ is a robust term.

The TDE technology can be used to estimate the unknown terms $(\Delta P)$ as follows:

$$
\Delta \hat{P}=\Delta P_{(t-L)}
$$

where $L$ is a delay parameter whose value is an integer multiple of the sampling period, and $\Delta P_{(t-L)}$, represents the value of the unknown term before time $L$. The calculation process can be expressed as follows:

$$
\Delta P_{(t-L)}=\ddot{x}_{(t-L)}+\frac{b}{m} \dot{x}_{(t-L)}+\frac{k_{e}}{m} x_{(t-L)}-\frac{k_{e} d}{m} u_{(t-L)} .
$$

Control law u can be obtained by substituting Eq. (6) into Eq. (8): 


$$
\underbrace{u=\frac{m}{k_{e} d}\left(\ddot{x}_{d}+\frac{q}{p} K^{-1_{e} 2-p / q}+K_{s w} \operatorname{sgn}(s)\right)+\frac{b}{k_{e} d} \dot{x}+\frac{1}{d} x}_{\text {NTSM }}-\underbrace{\frac{m}{k_{e} d}\left(x_{(t-L)}+\frac{b}{m} x_{(t-L)}+\frac{k_{e}}{m} x_{(t-L)}-\frac{k_{e} d}{m} u_{(t-L)}\right)}_{\text {TDE }}(9)
$$

Remark 1. Equation (9) shows that TDE aims to estimate the system dynamics at the current moment from the system state at the previous moment to achieve an accurate compensation for the system dynamics. This approach can ensure that the required gain of the NTSM item is greatly reduced, and the system robustness is improved while the quality is guaranteed.

Remark 2. Equation (9) shows that the sliding mode surface is the continuous and differentiable NTSM, and the TDE technology realizes the estimation of the unknown items and completes its compensation. The design of the NTSM and TDE terms are model-based. The proposed controller has a higher control accuracy compared with the model-free control.

Sign function $u \operatorname{sgn}(s)$ in the control law leads to chattering, of which the amplitude is $K_{s w}$. Saturation function $\operatorname{sat}(s, \varphi)$ is used instead of $\operatorname{sign}$ function $\operatorname{sgn}(s)$ to reduce chattering and smoothen the output of control law $u$ [22].

$$
\operatorname{sat}(s, \varphi)= \begin{cases}\frac{s}{|s|} & \text { if }|s| \geq \varphi \\ \frac{s}{\varphi} & \text { if }|s|<\varphi\end{cases}
$$

where $\varphi$ is the thickness of the boundary layer.

The structure of the proposed NTSM-TDE-RED controller is shown in Figure 2.

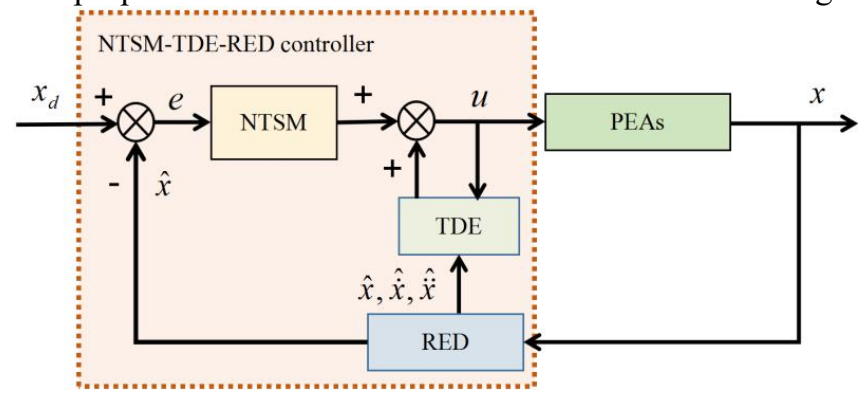

Fig. 2. Structural diagram of the NTSM-TDE-RED controller.

\subsection{Controller for comparison}

The proposed NTSM-TDE-RED controller is a model-based sliding mode controller. Based on the same design idea, the controller occupies a generalized model structure, and the controllers of other structures can be derived from it.

Controller 1 (Jin's controller). Reference [23] reported that a new positive parameter $\bar{M}$ is introduced, and a new unknown term $\mathrm{N}$ is defined. 


$$
N=\left(\frac{M}{k_{e} d}-\bar{M}\right) \ddot{x}+\frac{C}{k_{e} d} \dot{x}+\frac{x}{d}+\frac{h}{d}-\frac{f_{d}}{k_{e} d} .
$$

The dynamic model (1) is rewritten as follows:

$$
\bar{M} \ddot{x}+N=u .
$$

The unknown terms can be estimated by the TDE technology

$$
\hat{N}=N_{t-L}=u_{t-L}-\bar{M} \ddot{x}_{t-L} .
$$

A control law u without a model control can be obtained according to dynamic model (13) in combination with the design method named NTSM-TDE-RED controller.

$$
u_{\text {Jin }}=\underbrace{\bar{M}\left[\ddot{x}_{d}+\frac{q}{p} K^{-1_{e}^{2-p / q}}+K_{s w} \operatorname{sgn}(s)\right]}_{\text {NTSM }}+\underbrace{u_{t-L}-\bar{M} \ddot{x}_{t-L}}_{T D E}
$$

The above control scheme was first designed by Jin [23].

Controller 2 (Hsia's controller). If the NTSM term in Jin's controller is replaced by a proportional-derivative $(\mathrm{PD})$ term, then the controller is evolved into an PD controller on the basis of the TDE technology [24]. In dynamic model (13), this study takes

$$
u_{H i s a}=\underbrace{\bar{M}\left[\ddot{x}_{d}+K_{p} e+K_{D} \dot{e}\right]}_{P D}+\underbrace{\Delta \hat{N}}_{T D E} .
$$

In combination with the TDE technology, control law $\mathrm{u}$ is designed as follows:

$$
u_{H i s a}=\underbrace{\bar{M}\left[\ddot{x}_{d}+K_{p} e+K_{D} \dot{e}\right]}_{P D}+\underbrace{u_{t-L}-\bar{M} \ddot{x}_{t-L}}_{T D E} .
$$

The time-delay estimate error in Jin's and Hsia's control schemes is defined as follows:

$$
\Delta \widetilde{N}=\Delta N-\Delta \hat{N}
$$

Remark 3. Jin's and Hsia's control schemes contain $\dot{e}$ items. The estimated speed value obtained by the RED technology still includes certain errors. Accordingly, the value of $\bar{M}$ should be reduced to minimize the effect of errors on the control quality.

Remark 4. Jin's and Hsia's controllers are based on the model-free control, and their dynamic equations are highly concise. These controllers have a simpler structure, smaller calculation volume, and easier engineering implementation than NTSM-TDE-RED controllers. However, the NTSM-TDE-RED controller has high control accuracy. Each controller has its own strengths.

\section{Controller stability analysis}


The closed-loop stability analysis of the NTSM-TDE-RED controller is completed in this section. The TDE error $\Delta \widetilde{P}=\Delta P-\Delta \hat{P}$ is defined, and a positive number $\phi$ makes $\Delta \widetilde{P}$ bounded, that is, $|\Delta \widetilde{P}| \leq \phi$. The proof of the boundedness of the error $\Delta \widetilde{P}$ is shown in reference [19]. The displacement error equation of the closed-loop system can be obtained by substituting Eq. (9) into simplified dynamic model (4) as follows:

$$
\ddot{e}=\Delta \widetilde{P}-\frac{q}{p} K^{1^{2} 2-p / q}-K_{s w} \operatorname{sgn}(s) .
$$

Remark 5. The displacement error equation of the closed-loop system shows that displacement error $e$ is independent of the desired displacement and external disturbance. This condition enables convergence speed of the displacement error e to be unaffected by external disturbance during the actual operation of the controller. Moreover, this condition fully demonstrates that the NTSM-TDE-RED controller has strong robustness.

The stability proof process of the closed-loop system is given in detail as follows:

Lyapunov function [24] is defined as follows:

$$
V=\frac{1}{2} s^{2}
$$

This function is differentiated with time, thereby resulting in

$$
\begin{aligned}
\dot{V} & =s \dot{s}=s\left[\dot{e}+\frac{p}{q} K \dot{e}^{p / q-1_{\dot{e}}}\right]=s\left\{\dot{e}+\frac{p}{q} K \dot{e}^{p / q-1} \times\left[\Delta \widetilde{p}-\frac{p}{q} K^{-1_{\dot{e}}^{2-p / x}}-K_{s w} \operatorname{sgn}(s)\right]\right\} \\
& =\frac{p}{q} K \dot{e}^{p / q-1} \times\left(-|s| K_{s w}+s \Delta \widetilde{P}\right) .
\end{aligned}
$$

This study obtains $K_{s w} \geq|\Delta \widetilde{P}|$. Then, $|s| K_{s w} \geq|s||\Delta \widetilde{P}| \geq s|\Delta \widetilde{P}|$. Therefore, $\dot{V} \leq 0$.

The convergence analysis is as follows: according to LaSalle's invariant set theorem [25], when $t \rightarrow \infty, s \rightarrow 0$, that is, $e \rightarrow 0, \dot{e} \rightarrow 0$.

\section{Computer simulation experiment}

A computer simulation experiment is performed to verify the effectiveness of the proposed controller. MATLAB/Simulink is used as the simulation environment [26], and the simulation step of $0.1 \mathrm{~ms}$ is selected. The proposed control strategy is compared with Jin's and Hsia's control schemes to verify the effectiveness of the proposed controller.

The control system achieves linearization of the input and output by accurately tracking the desired displacement. Traditional root mean square error (RMSE) and maximum error (ME) [27] are used to evaluate the different controllers. Thereafter, the characteristics and performance of each controller can be quantitatively compared. 


$$
\begin{aligned}
& R M S E=\sqrt{\sum_{i=1}^{N}\left(x_{d i}-x_{i}\right)^{2} / N_{1},} \\
& M E=\max \left(\left|x_{d i}-x_{i}\right|\right)
\end{aligned}
$$

where NI represents the total number of sampling points; and xdi and xi are the expected and actual displacements of the i sampling points, respectively.

The parameters of the controller were adjusted in an ascending order by trial-and-error method. The selected parameters are shown in Table 1 by comprehensively considering the chattering degree, RMSE, and ME.

Table 1. Parameter setting of the controllers.

Controllers

NTSM-TDE-RED

PD-TDE-RED

\section{Parameter value}

$$
\bar{M}=0.1, p=1.5, q=1, K=0.004, K_{s w}=105
$$

$\bar{M}=0.1, K_{P}=104, K_{D}=105$

We select a sinusoidal signal with variable frequency and amplitude as the desired trajectory and express it as follows to obtain representative simulation results:

$$
y=\left(4-4 \cos \left(4 \pi t e^{-0.12 t}\right)\right) \times e^{-0.2 t}
$$

Figure 3(a) shows that all three controllers can track the sine signal of the variable frequency and amplitude. Figure 3(b) indicates that the displacement error curves generated by the three controllers synchronously fluctuate with the desired trajectory signal. Table 2 shows that the control accuracy of the NTSM-TDE-RED controller is the highest, whereas that of Hsia's controller is the lowest. The table reports that the error analysis shows that the RMSE of the NTSM-TDE-RED controller is approximately $24.75 \%$ lower than that of Jin's controller. The ME is also reduced by approximately $25.01 \%$. By contrast, the output of Jin's controller has a certain degree of chattering. This phenomenon is due to the absence of a sign function in Hsia's controller. The ideal situation is the absence of chattering. The chatter of Jin's controller comes from the sign function. Such controller needs to set a large gain in its NTSM term Ksw because it is a model-free controller. The NTSM-TDE-RED controller is a model-based controller with a low proportion of unknown terms (Eq. [17]). Amplitude Ksw of the NTSM-TDE-RED controller can be smaller than that of Jin's controller to ensure that its output chatter is significantly reduced. The amplitude of the control law $u$ of Hsia's controller is larger than that of NTSM-TDE-RED and Jin's controller. This condition results in great energy consumption of Hsia's controller.

Table 2. Control accuracy of the controllers.

\begin{tabular}{lll}
\hline Controllers & $R M S E$ & $M E$ \\
\hline Hsia's controller & 0.2816 & 0.7894 \\
Jin's controller & 0.0598 & 0.2023 \\
NTSM-TDE-RED & 0.0450 & 0.1517 \\
\hline
\end{tabular}




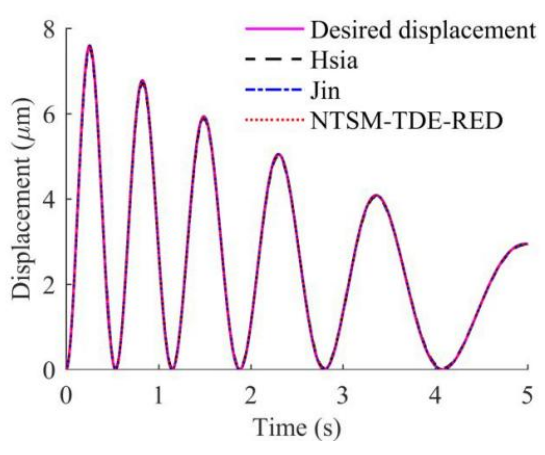

(a)

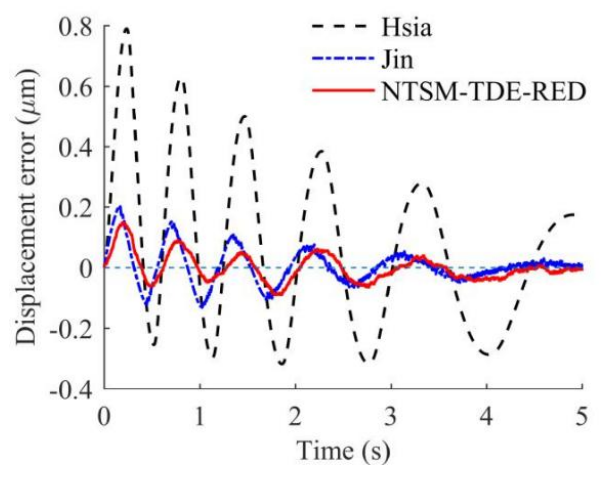

(b)

Fig. 3. Performance curves of controllers when tracking fluctuation signals. (a) Displacement, and (b) displacement error.

The NTSM-TDE-RED controller exhibits a high control accuracy and excellent quality of energy conservation when tracking continuous and differentiable fluctuating signals.

The role of the TDE technology in the controller is further examined, and the proportion of the TDE term in the total control law is evaluated. Figure 4 shows that the total control law $\mathrm{u}$ is indicated by a solid red line, and the output of the TDE term UTDE is denoted by a blue dotted line. Figures 4(a) and 4(b) demonstrate that the output u of Hsia and Jin's controllers heavily relies on the TDE technology.

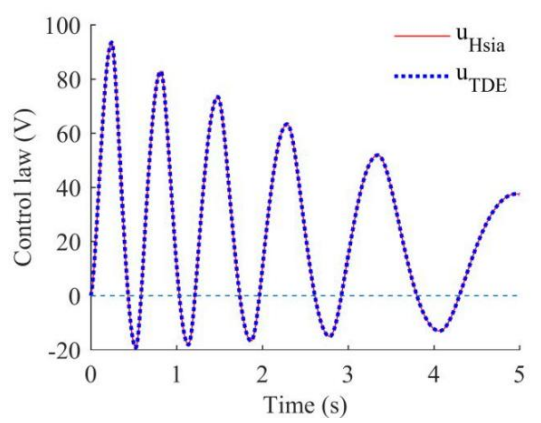

(a)

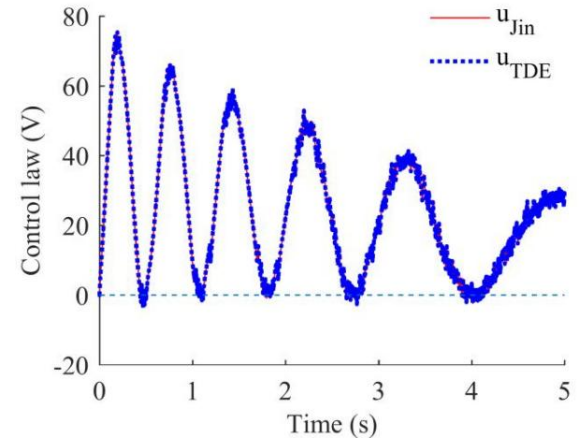

(b)

Figure 4. Control law $u$ and TDE output curves of the three controllers. (a) Control law uHsia and TDE output curve of Hsia's controller, (b) control law uJin and TDE output curve of Jin's controller.

Remark 6. In model-free controllers (for example, Hsia and Jin's controllers), the value of $\bar{M}$ needs to be reduced to control the influence of the acceleration estimation error in RED to achieve high control accuracy. The control law heavily depends on the TDE technology. The NTSM TDE-RED controller fully utilizes the functions of NTSM and TDE. These two technologies complement each other, thereby achieving the purpose of the controller design. Specifically, the NTSM term is used to implement a robust control, and the TDE technique is utilized to implement estimation and compensation of the unknown terms. 


\section{Semiphysical simulation experiment}

In this section, the experimental system of PEAs is constructed on the basis of the XPC semiphysical simulation environment [28]. The model of PEAs is Pst120/7/20VS12, and that of the voltage amplifier is E00.6, which are both produced by Harbin Coremorrow Co. Ltd. The displacement signal is collected by a LK-H020 laser displacement sensor (Keyence (China) Co. Ltd.) with a measurement accuracy of $0.02 \mu \mathrm{m}$.

The control algorithm operated on the host machine is transmitted to the target machine after compilation. The digital signal output by the target machine is amplified by a voltage amplifier after digital-to-analog conversion, and then PEAs are driven to move. The displacement signal collected by the laser displacement sensor is inputted to the target machine after the analog-to-digital conversion.

In the semiphysical simulation experiment, we select the sine signal with a variable frequency and an amplitude used in the previous section as the desired trajectory to establish comparability with the computer simulation experiment. The experimental results are shown in Figure 6. The semiphysical simulation experiments show that the trajectory tracking accuracy is much lower than that of computer simulation experiments. This phenomenon is due to environmental disturbances, measurement noise effects, and limitations of the laser displacement sensor acquisition accuracy. However, the three controllers can still successfully implement the trajectory tracking process. The RMSES obtained by the three controllers are $0.21,0.14$, and $0.1 \mu \mathrm{m}$, and the $M E$ are $0.62,0.31$, and $0.23 \mu \mathrm{m}$ respectively. The control precision of the NTSM-TDE-RED controller is high.

\section{Conclusion}

We propose a high-precision robust controller that combines the NTSM, TDE, and RED technologies to overcome the uncertain nonlinear factors, such as hysteresis, creep, and high-frequency vibration, that are common in trajectory tracking of PEAs. The NTSM is used to make the controller robust, and the RED technology is utilized to achieve an accurate estimation of the full state. Comparative experiments of the computer and semiphysical simulations prove that the proposed controller can effectively overcome the influence of disturbances and accurately control PEAs to achieve accurate trajectory tracking. The RMSE and ME reached 0.11 and $0.23 \mu \mathrm{m}$, respectively. Therefore, the proposed NTSM-TDE-RED controller has strong engineering applicability and can be widely used in the precision motion control of PEAs.

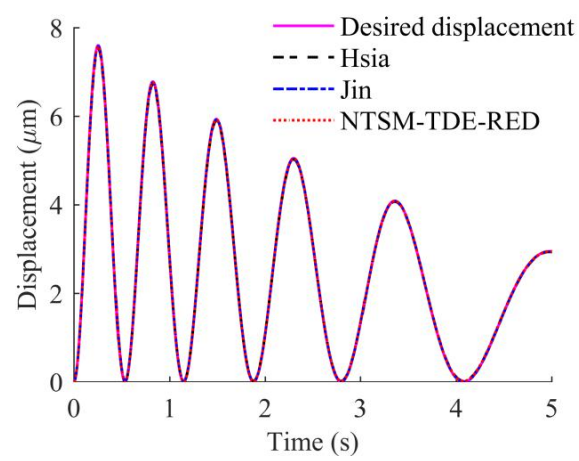

(a)

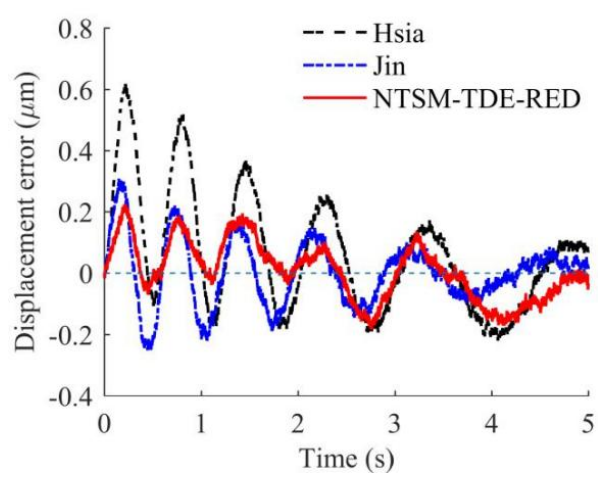

(b) 
Fig. 6. Performance curve of controller when tracking fluctuation signals. (a) Displacement, and (b) error.

This research is supported by the Independent Application Project Social Development Scientific Research of Hangzhou Science and Technology Commission: (Grant No. 20191203B30), Zhejiang Public Welfare Technology Application Research Support Project: (Grant No. LGG20E050012), Wenzhou Basic Scientific Research Project: (Grant No. G20190012).

\section{References}

1. $\mathrm{Wu} \mathrm{Z}$ and $\mathrm{Xu} \mathrm{Q}$, Survey on recent designs of compliant micro-/nano-positioning stages. Actuators, 2018;7 (1): 5.

2. Yu S., Ma J., Wu H., and Kang S., Robust precision motion control of piezoelectric actuators using fast nonsingular terminal sliding mode with time delay estimation. Measurement and Control, 2019; 52(1-2): 11-19.

3. Wang Z, Liu L, Wang Y. Stable nanomanipulation using atomic force microscopy: A virtual nanohand for a robotic nanomanipulation system. IEEE Nanotechnology Magazine, 2013; 7(4), 6-11.

4. Gozen B.A. and Ozdoganlar O.B. Design and evaluation of a mechanical nanomanufacturing system for nanomilling. Precision Engineering, 2012; 36(1): 19-30.

5. Ghosh B., Jain R.K., Majumder S., Roy S., and Mukhopadhyay S. Experimental characterizations of bimorph piezoelectric actuator for robotic assembly. JIMSS, 2017; 28(15): 2095-2109.

6. Putra A.S., Huang S., Tan K.K., Panda S.K., and Lee T.H. Design, modeling, and control of piezoelectric actuators for intracytoplasmic sperm injection. IEEE Transactions on Control Systems Technology, 2017; 15(5): 879-890.

7. Jiang H., Ji H., Qiu J., and Chen Y. A modified prandtl-ishlinskii model for modeling asymmetric hysteresis of piezoelectric actuators. IEEE Transactions on Ultrasonics, Ferroelectrics, and Frequency Control, 2010; 57(5): 1200-1210.

8. Rakotondrabe M. Bouc-Wen modeling and inverse multiplicative structure to compensate hysteresis nonlinearity in piezoelectric actuators. IEEE Transactions on Automation Science and Engineering, 2010; 8(2): 428-431.

9. Ms A., As B., Hysteresis compensation-based robust output feedback control for longstroke piezoelectric actuators at high frequency. Sensors and Actuators A: Physical, 2021

10. K. Hergli, H. Marouani, M. Zidi, Y. Fouad, and M. Elshazly, Identification of Preisach hysteresis model parameters using genetic algorithms, Journal of King Saud University-Science, 2019; 31(4): 746-752.

11. Opreni A., Boni N., Analysis of the Nonlinear Response of Piezo-Micromirrors with the Harmonic Balance Method. Actuators, 2021; 10(2): 21-30.

12. Oh J. and Bernstein D.S., Semilinear Duhem model for rate-independent and ratedependent hysteresis, ITAC, 2005; 50(5): 631-645.

13. Huang L, Li Y, Tong S. Fuzzy Adaptive Output Feedback Control for MIMO Switched Nontriangular Structure Nonlinear Systems With Unknown Control Directions. IEEE Transactions on Systems, Man, and Cybernetics: Systems, 2020, 50(2):550-564. 
14. Xie MY, Yu SD, Lin HP,Wu HT. Improved Sliding Mode Control with Time Delay Estimation for Motion Tracking of Cell Puncture Mechanism. IEEE Transactions on Circuits and Systems I, 2020. (DOI: 10.1109/TCSI.2020.2981629).

15. Yu, S., Xie, M., Wu, H., Ma, J., Li, Y., \& Gu, H. Composite proportional-integral sliding mode control with feedforward control for cell puncture mechanism with piezoelectric actuation. ISA Transactions; 2020, (DOI: 10.1016/j.isatra.2020.02.015).

16. Yu S., Xie M., Wu H., et al., Design and control of a piezoactuated microfeed mechanism for cell injection. The International Journal of Advanced Manufacturing Technology, 2019; 105(12): 4941-4952.

17. Kamal S., Moreno J.A., Chalanga A., Bandyopadhyay B., and Fridman L.M. Continuous terminal sliding-mode controller. 2016; 69: 308-314.

18. Asl R.M., Hagh Y.S., and Palm R. Robust control by adaptive non-singular terminal sliding mode. Engineering Applications of Artificial Intelligence, 2017; 59: 205-217.

19. Jin M., Lee J., and Ahn K.K. Continuous nonsingular terminal sliding-mode control of shape memory alloy actuators using time delay estimation. IEEE/ASME Transactions on Mechatronics, 2014; 20(2): 899-909.

20. Lee J., Chang P.H., and Jin M. Adaptive integral sliding mode control with time-delay estimation for robot manipulators. ITIE, 2017; 64(8): 6796-6804.

21. Levant A. Higher-order sliding modes, differentiation and output-feedback control. IJC, 2003; 76(9-10): 924-941.

22. Zhao X., Yang H., XiaW., andWang X. Adaptive fuzzy hierarchical sliding-mode control for a class of MIMO nonlinear time-delay systems with input saturation, IEEE Transactions on Fuzzy Systems, 2016; 25(5): 1062-1077.

23. Jin M., Lee J., Chang P.H., and Choi C. Practical nonsingular terminal sliding-mode control of robot manipulators for high-accuracy tracking control, ITIE, 2009; 56(9): 3593-3601.

24. Shtessel Y.B., Moreno J.A., and Fridman L.M. Twisting sliding mode control with adaptation: Lyapunov design, methodology and application, Autom, 2017; 75: 229-235.

25. Hespanha J.P. Uniform stability of switched linear systems: Extensions of LaSalle's invariance principle, ITAC, 2004; 49(4): 470-482.

26. Karner T., Gotlih J. Position Control of the Dielectric Elastomer Actuator Based on Fractional Derivatives in Modelling and Control. Actuators, 2021; 10(1):184.

27. Yu SD, Wu HT, Xie MY, Ma JY, Precise Robust Motion Control of Cell Puncture Mechanism Driven by Piezoelectric Actuators with Fractional-order Nonsingular Terminal Sliding Mode Control, Bio-Design and Manufacturing, 2020; (DOI: 10.1016/j.isatra.2020.02.015)

28. Chin C., Lum S. Rapid modeling and control systems prototyping of a marine robotic vehicle with model uncertainties using xPC Target system, OcEng, 2011; 38(17-18): 2128-2141. 